\title{
An Implementation of Effective Logo Matching and Detection using Multiple Descriptors to Enhance the Resolution
}

\author{
Poornaiah Billa, PhD \\ Associate Professor in EIE Dept. \\ VNR Vignana Jyothi Inst.of Engg\& \\ Tech.Nizampet, \\ Hyderabad, TS, India-500090
}

\author{
Ashok Kumar Balijepalli \\ Assistant Professor in ECE Dept. \\ Universal College of Engg \& Tech. \\ Perecherla,Guntur, \\ A P,India 522438
}

\author{
I. RamaKoteswara Rao \\ Assistant Professor in ECE Dept. \\ Universal College of Engg \& Tech. \\ Perecherla, Guntur, A P, India- \\ 522438
}

\begin{abstract}
In current trends the logos are playing a vital role in industrial and all commercial applications. Fundamentally the logo is defined as it's a graphic entity which contains colors textures, shapes and text etc., which is organized in some special visible format. But unfortunately it is very difficult thing to save their brand logos from duplicates. In practical world there are several systems available for logo reorganization and detection with different kinds of requirements. In some partial occlusions it should be robust to transfer the large range of photometric and geometric features of a logo which they are not captured in isolation. Two dimensional global descriptors are used for logo matching and reorganization. The concept of Shape descriptors based on Shape context and the global descriptors are based on the logo contours. There is an algorithm which is implemented for logo detection is based on partial spatial context and spatial spectral saliency (SSS). The SSS is able to keep away from the confusion effect of background and also speed up the process of logo detection. All such methods are useful only when the logo is visible completely without noise and not subjected to change. These types of methods are not suitable for practical images where insufficient resolution is the drawback of these methods. To overcome these drawbacks we proposed a multiple descriptors method along with context dependent similarity concept. The multiple descriptors are scale invariant feature transform (SIFT), Speeded up robust feature (SURF), histogram oriented gradient (HOG) and Gradient location and orientation histogram (GLOH). By using this method we assure high resolution and great accuracy.
\end{abstract}

\section{Keywords}

Logo Matching and Recognition, Context Dependent Similarity, Scale Invariant Feature Transform (SIFT), Speeded Up Robust Feature (SURF), Histogram Oriented Gradient (HOG) and Gradient Location and Orientation Histogram (GLOH).

\section{INTRODUCTION}

Logo sometimes also known as trademark have high importance in today's marketing world, because it carries the goodwill of the company and the product. Logo recognition has been specifically used in application areas such as enterprise identification, entertainment advertising, vehicle recognition, road sign reading, and website summarization by image content based image retrieval. For a particular logo recognition system, features related to visual contents are first extracted to describe the logo images. Then a similarity measure is defined to compare the query image with the target images which are saved in a logo database using the extracted features. After that, a target logo most similar to the query image is retrieved. Since the query logo image may be taken by a hand held mobile phone-camera operating at varying different viewpoints under different lighting environments. The query image may differ substantially from the database target image due to geometric transformations like viewpoint, rotation, and scaling changes and photometric transformation like lighting, noise, and image blur.
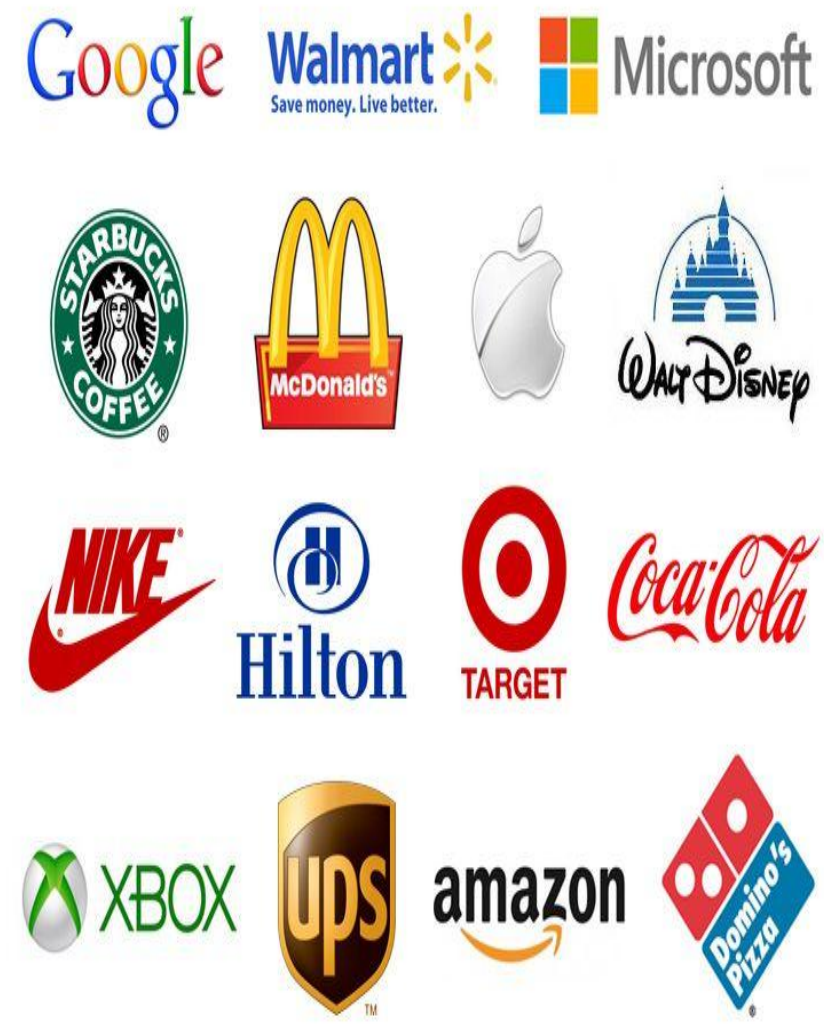

Fig 1: Some most popular sample logos

Therefore it is a challenge to the logo recognition system to extract the features robust to the above inevitable imaging variations. A logo is nothing but a graphic entity containing colors, shapes, textures, and perhaps text as well, organized in particular spatial layout format. Color features are often easily obtained from the logo image. The color histogram is probably one of the most popular gross representations of the foreground object in which the precise spatial information is lost, so an extraction is generally impossible. Since logo is designed with a setting of few color combinations, so color 
will be ignored as far as the unique identity of a $\log$ which is represented as an intrinsic graphic pattern. On the other hand, the text in the logo is often modified to add to its aesthetic appealing; its segmentation or the OCR processing may not be easy and also unnecessary for the logo handled by a shape analyzer. Similarly, if a logo contains texture patterns, the texture patterns can be treated as a graphic pattern and handled with other parts together. Hence, shape analysis of the logo is the main concern here. In this study, experiments on logo images are conducted with three existing techniques called as Scale Invariant Feature Transform (SIFT), Speeded Up Robust Feature (SURF), Histogram Oriented Gradient (HOG) and Gradient Location and Orientation Histogram (GLOH). Finally, the Manhattan distance classifier is used to measure the similarities between the samples of training logo images and testing logo images of various classes.

\section{LITERATURE SURVEY}

Previously there are several contributions made by eminent people for $\log 0$ matching and recognition. Apostolos P. Psyllos, Christos-Nikolas E. Anagnostopoulos proposed a SIFT-based enhancement matching scheme algorithm for vehicle logo recognition. This algorithm is assessed on a set of nearly 1200 logo images that belong to ten distinctive vehicle manufacturers. It is shown that the proposed enhanced matching approach boosts the recognition rate in vehicle logos making it suitable for real-time applications. In all the previous methods they provided excellent results for image or logo matching and detection. But the multiple descriptor methods are also used previously for the fake logo detection.

But in this paper we propose enhancement of the image resolution along with accuracy. Smita A. Patil and T.B. Mohite Patil proposed a solution for logo recognition based on context dependent similarity that directly incorporates the spatial contact of local features. It is suitable to detect similarities and differences between both nearer and duplicate $\operatorname{logos}$ by intensity matching. The solution is proved to be highly effective and responds to the requirement of logo detection and recognition in real world images. Ke Gao, Shouxun Linl, Yongdong Zhang, Sheng Tang, Dongming Zhang collected a data set of 10,016 images from the web and TV shows. Ten popular logos are selected for testing such as Starbucks, Coca-Cola, Nike, CNN, etc. These logos are either on products or in cluttered background, and very different in position, orientation, and sizes. Related images for each logo category vary from 30 to 200 , and the rest are non-related images also contained as interference.

The proposed scheme is designed to speed up logo localization and improve the detection precision at the same time. Chinmoy Biswas, Joydeep Mukherjee contributed for the logo detection and accuracy. They also proposed an algorithm and the comparative statement of SIFT, SURF and HOG descriptors.

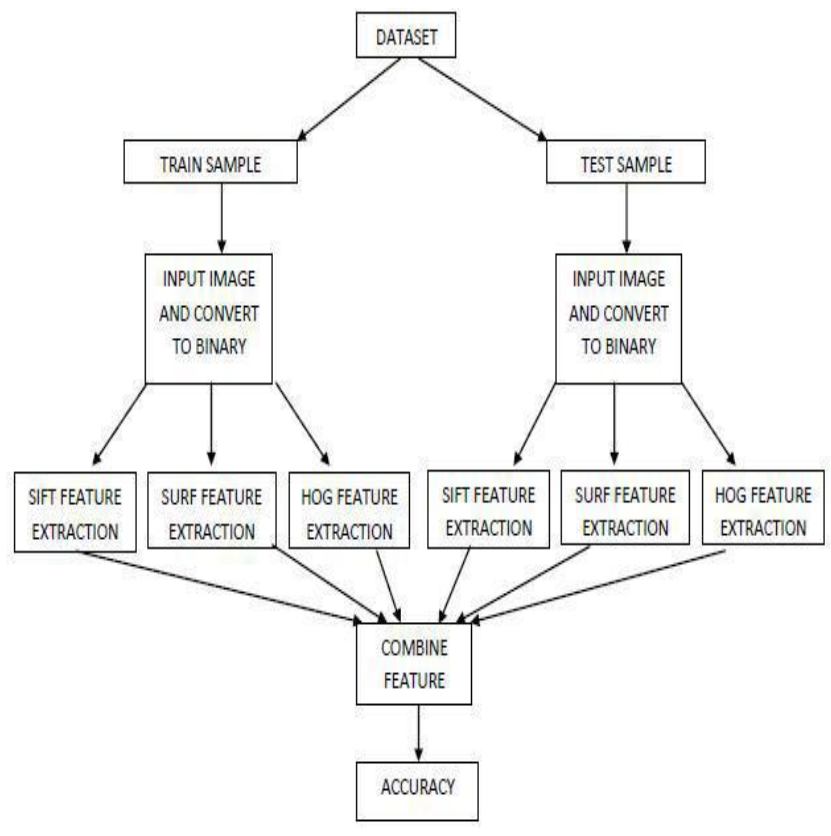

Fig 2. Existed Method for logo matching and recognition

Ch. Divya Susmitha, L.Padmalatha , B. Suganya and A.C. Santha Sheela etc. authors are given several methods for effective matching and detection of logos.

\section{PROPOSED METHOD}

In this work we proposed multiple descriptors method along with context dependent similarity for enhancing the accuracy and resolution of the logo. This approach for logo recognition and detection depends on the description of a context dependent similarity (CDS) kernel. This method is used for descriptors and is not limited to any derivable arrangement model. It is mainly deals with the spatial context of local features and the scheme of recognition process. The data set is obtained from google for sample testing. All these logos are in TIFF format and resized to $150 \times 150$ pixels, converting images into binary image and into double.

\section{A. Scale Invariant Feature Transform descriptors:}

The SIFT descriptors are computed for normalized image patches with the code provided by Lowe. A is a $3 \mathrm{D}$ histogram of gradient location and orientation. The resulting descriptors are of dimension 128 .

Working with SIFT Descriptors: One image yields

- $\mathrm{N}=128$ - dimensional description: each one is a histogram of the gradient orientations within a patch [n*128 matrix]

- N- Scale parameters specifying the size of each patch

[n*1 vector ]

- N-Orient parameters specifying the angle of the patch

[n*1 vector]

- $\quad \mathrm{N}-2 \mathrm{D}$ points giving positions of the patches $[\mathrm{n} * 2$ matrix] 


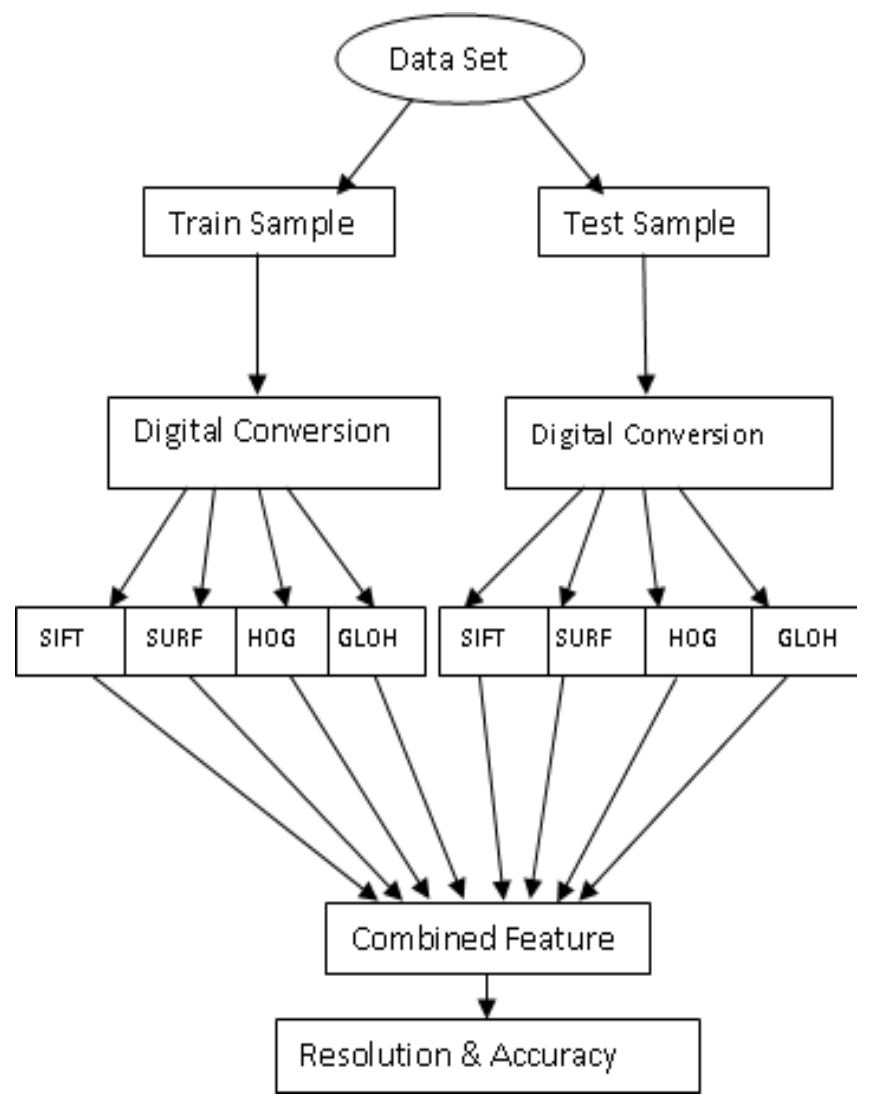

Fig 3: Proposed Method

\section{B. Speeded Up Robust Feature:}

The SURF, when compared with the best performance of SHIFT to other descriptors are remarkable. Information localized crudely for mixing and in terms of scale or space the distribution of gradient related features seems to yield good distinctive power, while fending off the effects of localization errors. Gradients reduce the effect of photometric changes by using relative strengths and orientations. The similar properties based on proposed SURF descriptors are even further with a complexity strip down. The information from a circular region around the interest point is based on first step.

- $\quad \mathrm{AB}=\operatorname{nor}(\mathrm{D} 1(\mathrm{X},)$.

- $\mathrm{D} 3(1, \mathrm{x})=\mathrm{AB}$; where $\mathrm{x}=64$;

- $\mathrm{D} 3(1, \mathrm{x})=\mathrm{AB}$; where $\mathrm{x}=64$;

\section{Histogram Oriented Gradient:}

The purpose of object detection in computer vision and image processing is the histogram of oriented gradients is a feature descriptors used. This technique counts occurrences of gradients orientation in localized portions of an image. This technique is similar to scale invariant, edge orientation, feature transform descriptors, shape contexts computed on a dense grid of uniformly spaced cells,. And other uses are overlapping of local contrast normalization for improved accuracy.

\section{Gradient Location and Orientation Histogram:}

The GLOH is an extension of SIFT descriptor and is a gradient location orientation histogram to increase its robustness and distinctive for logo polar location grid. 8 in angular direction and 3 bins in radial direction and will not divide in angular directions of the central bin. In 16 bins the gradient orientations are quantized, a 272 bin histogram it gives. With Principle Component Analysis (PCA) the size of this descriptors were reduced. It is estimated on 47000 image patches collected from various images by the covariance matrix for PCA. For descriptors 128 largest eigenvectors are used.

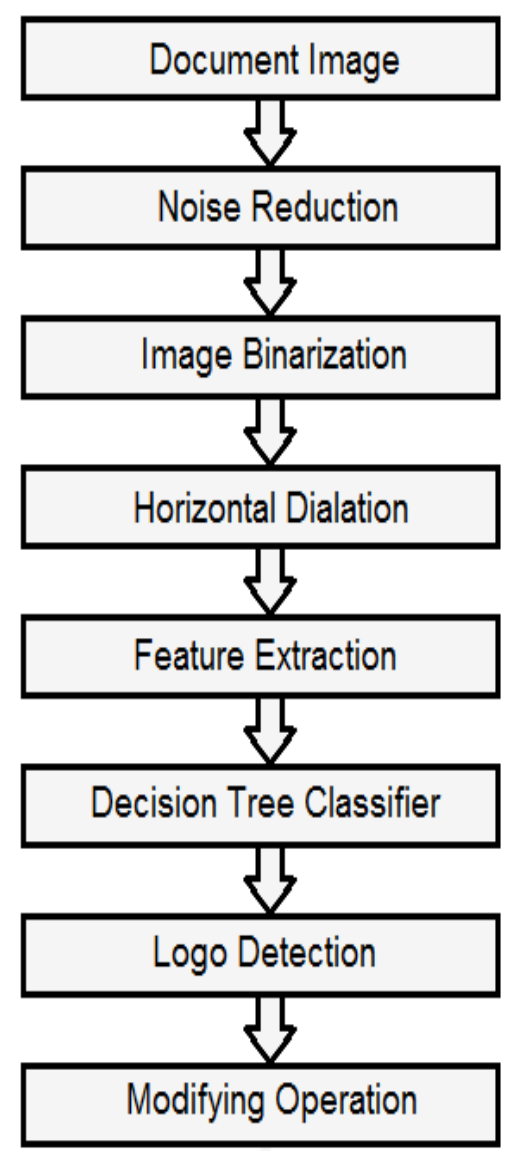

Fig 4: Algorithm for Logo Recognition

Totally 25 classes have taken in this method and 5 images are used to form a training set per class. Resized to $150 \times 150$ pixels, converted binary image, SIFT descriptors and HOG descriptors of all the images and they are calculated for first 5 images which belong to a particular class and is where $128+65+81=274$ is the feature vector size.

\section{RESULTS AND CONCLUSION}

By using MATLAB tools this method is implemented. This is a novel logo detection and localization approach based on a new class of similarities referred to as context dependent. In several aspects the proposed system strength resides. So many numbers of popular logos were collected for the implementation of this method and formed as data set with the particular name. We have chosen an image during execution process and then select another logo which is need to compare pixel intensity values with another logo. Between these logos we find more compatibility. Finally justifying the logo is original one or not. The following table describes the percentage of accuracy got with different process. And the graph shows precession and recall values. 
Logo Matching with Different Descriptor Combination:

Table 1: The Comparison Sample Result

\begin{tabular}{|l|l|}
\hline Process & Percentage Accuracy \\
\hline Only SIFT & $56.66 \%$ \\
\hline Only HOG & $76.66 \%$ \\
\hline Only SURF & $81.66 \%$ \\
\hline SIFT with HOG & $73.33 \%$ \\
\hline SIFT with SURF & $83.33 \%$ \\
\hline SURF with HOG & $85.00 \%$ \\
\hline SIFT , HOG and SURF & $91.60 \%$ \\
\hline GLOH & $96.66 \%$ \\
\hline
\end{tabular}

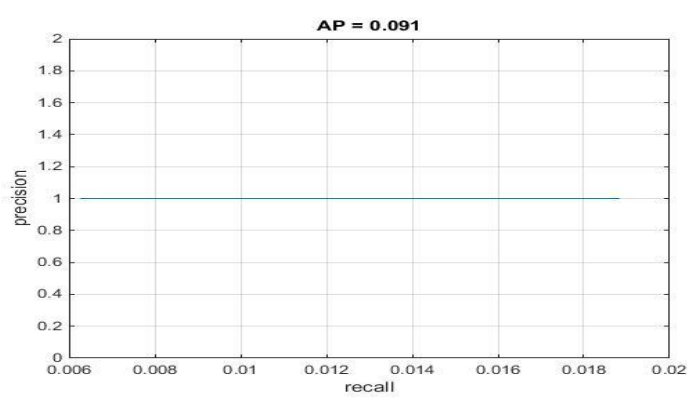

Fig 6: The graph between precision Vs Recall

\section{REFERENCES}

[1]. Chinmoy Biswas,Joydeep Mukherjee "Logo Recognition Technique using Sift Descriptor, Surf Descriptor and Hog Descriptor" International Journal of Computer Applications (0975 - 8887) Volume 117 No. 22, May 2015.

[2]. Ch.Divya Susmitha, L.Padmalatha "Context Dependent Logo Detection and Recognition based on Context Dependent Similarity" Kernel International Journal of Computer Applications (0975 - 8887) Volume 106 No.11, November 2014.

[3]. Apostolos p. Psyllos, christos-nikolaos e. Anagnostopoulos "vehicle logo recognition using a siftbased Enhanced matching scheme" IEEE transactions on intelligent transportation systems, vol. 11, no. 2, june 2010.

[4]. Shu-Kuo Sun And Zen Chen "Robust Logo Recognition For Mobile Phone Applications"Journal Of Information Science And Engineering 27, 545-559 (2011).

[5]. Ke gao , shouxun lin1, yongdong zhang, sheng tang, dongming zhang "logo detection based on spatialspectral saliency and Partial spatial context" national basic research Program of china (973 program, 2007cb311100),National high technology and research development Program of china (863 program, 2007aa01z416).

[6]. B.Suganya and A.C.Santha Sheela"Finding Fake Logo Using CDS Logo Detection And Recognition Algorithm" International Journal of Scientific \& Engineering Research, Volume 5, Issue 3, March-2014.

[7]. Wenju Li and Ling Li, "A Novel Approach for Vehiclelogo Location Based on Edge Detection and Morphological Filter, 2nd International Symposium on Electronic Commerce and Security, Nanchang, vol: 1, pp. 343-345, 2009.

[8]. Nabeel Younus Khan,Brendan McCane,Geoff Wyvill "SIFT and SURF Performance Evaluation Against Various Image Deformations on Benchmark Dataset, International Conference on Digital Image Computing: Techniques and Applications,2011 ,PP.501-506.

[9]. Syed Yasser Arafat, Syed Afaq Husain, Iftikhar Azim Niaz and Muhammad Saleem, "Logo Detection and Recognition in Video Stream", 5th International Conference on Digital Information Management, Thunder Bay, Canada, pp. 163 - 168, 2010.

[10].Ma Y F, Zhang H J, "Contrast-based Image Attention Analysis By Using Fuzzy Growing. Proceedings of the $11^{\text {th }}$ ACM International Conference on Multimedia (MM2003). Berkeley, CA, USA: ACM, pp.374 - 381, 2003.

[11].Xiaodi Hou, Liqing Zhang, "Saliency Detection: A Spectral Residual Approach," Computer Vision and Pattern Recognition (CVPR), 2007.

[12].Herve Jegou, Matthijs Douze, and Cordelia Schmid, "Hamming Embedding and Weak Geometric Consistency For Large Scale Image Search,"ECCV, 2008.

[13].Sivic. J, Zisserman. A, "Video Google: A text retrieval approach to object matching in videos." In: ICCV. pp. 1470-1477.2003. 